\title{
Correction to: Supervisory Control of Discrete-Event Systems
}

\section{Correction to:}

\author{
W. M. Wonham and K. Cai \\ Supervisory Control of Discrete-Event Systems, \\ Communications and Control Engineering, \\ https://doi.org/10.1007/978-3-319-77452-7
}

The original version of the book was inadvertently published with incorrect information in Chapter 1, Chapter 2, Chapter 3, Chapter 4, Chapter 5, Chapter 6, and Chapter 9; the corrections have now been updated.

The updated version of these chapters can be found at https://doi.org/10.1007/978-3-319-77452-7 